\title{
Team Resource Management im OP und in der Endoskopie
}

\author{
Christian K. Lackner ${ }^{a, b}$ Heinzpeter Moecke ${ }^{c, d}$ Karin Burghofer \\ ${ }^{a}$ H:G I Hochschule für Gesundheit und Sport, Berlin, \\ ${ }^{\mathrm{b}}$ HealthCare Division, Drees und Sommer, München, \\ ${ }^{c}$ Hochschule für Angewandte Wissenschaften Hamburg (HAW) \\ ${ }^{\mathrm{d}}$ Asklepios Unternehmensbereich Medizin, Hamburg, \\ ${ }^{\mathrm{e}}$ Institut für Notfallmedizin und Medizinmanagement, Klinikum der Universität, München, Deutschland
}

\section{Schlüsselwörter}

Team Resource Management, TRM - Patientensicherheit . Risikomanagement · OP · Endoskopie · Sicherheitskultur

\section{Zusammenfassung}

Hintergrund: Klinischem Handeln ist implizit, dass die große Mehrzahl an risikoreichen und gleichzeitig prognoserelevanten Handlungen am Patienten nicht durch Einzelne erfolgt, sondern die Resultate einer Teamleistung (bisweilen ad hoc) sind. Methode: Publizierte Forschungsergebnisse zeigen nachvollziehbare Befunde, welche die Ursachen von Fehlermechanismen und -ketten darstellen und die Wirksamkeit von TRM(Team Resource Management)-Trainingsformaten belegen. Ergebnisse: Die Analyse der Literaturbefunde seit 2003 zeigt deutlich, dass die Ursache von prognose- oder zwischenfallrelevanten Fehlerketten in der klinischen Medizin zu mehr als $70 \%$ den Anwendern zuzuordnen ist. Die Implementierung und kontinuierliche Weiterentwicklung der Patientensicherheit im Bereich der Human Factors und die Anwendung der TRM-Leitsätze erfordert als Basis eine gute und breit akzeptierte Sicherheitskultur. Schlussfolgerungen: Initiativmaßnahmen wie regelmäßige realitätsnahe Simulationsteamtrainings, TRM-Formate und gut etablierte nichtpunitive Incident-Reporting-Systeme können hierbei effektiv zu einer stetigen und messbaren Verbesserung der Sicherheitskultur beitragen.

\section{Keywords}

Team Resource Management, TRM - Patient safety · Risk management · OR · Endoscopy - Safety culture

\section{Summary}

Team Resource Management in Surgery and Endoscopy Background: In the field of acute medicine, the vast majority of riskful and prognosis-relevant procedures are not performed by individuals but rather by (ad hoc) teams. Method: Findings in scientific papers impressively show the causes of medical mishaps and severe errors as well as the lasting effectiveness of training in team resource formats (Team Resource Management, TRM) in order to combat these chains of errors in acute medicine. Results: The analysis of the literature since 2003 and the numerous findings regarding the research of medical errors show that more than $70 \%$ of the severe and prognosis-relevant mishaps and complications can be assigned to the medical providers themselves. The implementation and continuous advancement of patient safety in the field of human factors as well as the application of TRM-related principles requires a sound and widely accepted safety culture as a basis. Conclusions: TRM training measures and non-punitive critical incident reporting systems effectively contribute to an increasing and measurable improvement of the safety culture in acute medicine.

\section{KARGER \\ Fax +497614520714 \\ Information@Karger.com}

www.karger.com (c) 2013 S. Karger GmbH, Freiburg

1662-6664/13/0293-0159\$38.00/0

Accessible online at:

www.karger.com/vim 


\section{Einleitung}

Während man noch vor Jahren Kliniker, die sich mit den Mechanismen der Fehlerentstehung und dem «Human Factor» beschäftigten, belächelt hat, ist die publizierte Erkenntnislage auch in der Medizin - wie in anderen Risikobereichen - erdrückend klar. Erkennt man diese methodisch sauber deduzierten Befunde der letzten 10 Jahre an, so ist zu konstatieren, dass die Ursache von prognose- oder zwischenfallrelevanten Fehlerketten in der klinischen Medizin zu mehr als $70 \%$ den Anwendern zuzuordnen ist (sogenannte «Human Factors»). Wenn Fehlerketten mithin wahrscheinlich vermeidbar wären, gewinnt das Team Resource Management (TRM) in solchen Handlungssektoren eine zentrale Bedeutung.

Es ist klinischem Handeln implizit, dass die große Mehrzahl an risikoreichen und gleichzeitig prognoserelevanten Handlungen am Patienten nicht durch Einzelne erfolgt, sondern die Resultate einer Teamleistung (bisweilen ad hoc) darstellen [1-7].

In diesem Kontext spiegelt sich in klinischen Führungsebenen nach wie vor die Haltung bzw. Annahme wider, dass eine Teamleistung dann erfolgreicher und nachhaltiger bezüglich der Aspekte der Patientensicherheit wäre, wenn alle Beteiligten «sich nur mal richtig anstrengen würden, um ein gutes Team zu sein» $[6,8]$.

Viele der sogenannten Risikoumfelder in anderen Handlungsbereichen beneiden die klinische Medizin um die grundsätzlich positive Haltung der Handelnden gegenüber der Aufgabenstellung - begleitet von einem überraschend hohen Maß an intrinsischer (!) Sicherheit. Dieser Umstand ist gleichermaßen wertvoll und bedeutend $[6,9,10]$.

Die hohe Motivation und positive Haltung existieren trotz der bisweilen widrigen institutionellen Rahmenbedingungen (z.B. hohe Arbeitsbelastung, wenig positive Rückmeldungen, steile Hierarchien) und einer noch immer in einigen Einrichtungen inhärenten sogenannten «Culture of Blame» (name $\rightarrow$ blame $\rightarrow$ shame) anstelle einer gemeinsamen Sicherheitskultur $[6,11]$.

\section{Fehlerentstehung und Fehlermechanismen}

Personal aus der Medizin und insbesondere der operativen Akutmedizin erfährt viele Belastungen, die mit denen in der Luft- und Raumfahrt vergleichbar sind (Tab. 1, Abb. 1). Medizinische Fehler sind vergleichbar multifaktoriell und fortschreitend, und menschliches Fehlverhalten (Human Error) ist ein wichtiger Faktor bei Fehlerketten [2, 8, 9, 12].

\section{Entstehung von Fehlern in der Akutmedizin}

Die Patientensicherheit wird vornehmlich durch die Leistungsfähigkeit des jeweiligen medizinischen Teams bestimmt. Fehler in der Medizin gehören zu den zehn häufigsten Todes-
Tab 1. Spezifika der operativen und interventionellen Akutmedizin (modifiziert nach [23])

\section{Hoher Komplexitätsgrad}

Akuter Handlungs- und Entscheidungsdruck

Interaktion zwischen Menschen, die sich in Stress befinden

Ungleichmäßige Arbeitsbelastung

Teilweise Adhoc-Teams, Zusammentreffen wenig eingespielter Teams Interdisziplinarität, unterschiedlicher Professionalisierungshintergrund (Präklinisch) teilweise schwierige Gegebenheiten, z.B. Lärm

Teilweise eingeschränkte diagnostische und therapeutische Möglichkeiten

Steile Hierarchien in der Medizin

Autoritäres Verhalten von Vorgesetzten

«Name blame shame»-Fehlerkultur

Hoher Technisierungsgrad

Ausbildung zumeist auf «technical skills» konzentriert

Große Anzahl an Schnittstellen und Patientenübergaben

Abb 1. Dimensionen des Team-ResourceTrainings in der Akutmedizin (aus [2], genehmigt SafeTrac ${ }^{\odot}$ Programm der DGU).

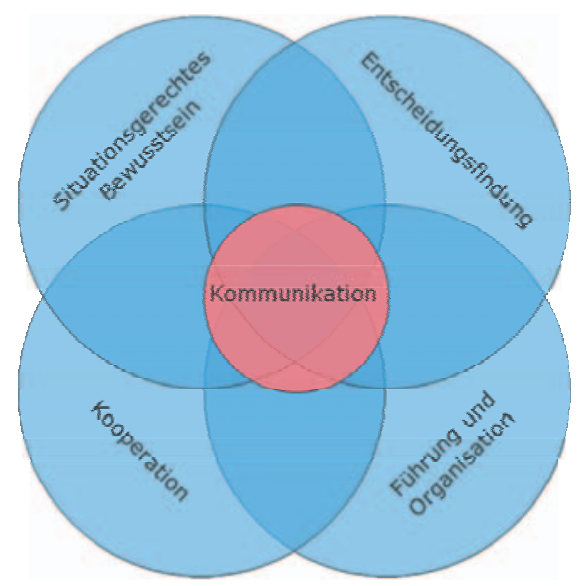

ursachen in Kliniken. Die meisten assoziierten Faktoren bei einer Zwischenfallentstehung sind menschliche Fehlleistungen wie Unterlassung einer Kontrolle, mangelnde situative Erfahrung, Vigilanzdefizit oder schlechte Kommunikation. Die akutmedizinische (Komplikations-)Behandlung ist stets ereignisabhängig und vernetzt.

Handlungsabläufe, die sich gegenseitig beeinflussen, laufen parallel ab. Das medizinische Team ist selbst Teil des Geschehens und kann viele Faktoren in der Akutsituation nicht beeinflussen $[2,8,9,12]$.

Der Patient als biologisches System ist komplex und nur bedingt einschätzbar. Dabei besteht oft ein situativ hoher Entscheidungs-, Handlungs- und Erwartungsdruck, was die Fehlertoleranz des Ablaufs weiter verringert. Nur durch eine «ergebnisorientierte Fehlerkultur» können die Mechanismen der Fehlerentstehung erforscht und Wege der Fehlervermeidung gefunden werden.

Jedes System - besonders ein so komplexes, wie es die interventionelle oder operative Medizin darstellt - beinhaltet sogenannte latente Fehler. Dies sind potenzielle Fehler bzw. Fehlermöglichkeiten, die innerhalb eines Systems «schlum- 
Kasten 1. TRM-Strategie «Dream Teams are made - not born!» (modifiziert nach [6])

Professionelle Leistungen auf höchstem Niveau können nur von Teams erwartet werden, die regelmäßig kritische Situationen im Team trainieren. Alles andere ist Glück und Zufall. Keine andere Disziplin oder Industrie würde ihre Teams so unvorbereitet in eigentlich vorhersehbare Zwischenfälle laufen lassen wie die Medizin. Menschliche Höchstleistungen kommen, egal in welchem Feld, ob Sport, Schach, Fliegerei oder Kunst, von jahrelangem intensivem Training. Trainiert werden dabei immer wieder die Dinge, die am wichtigsten sind und die am wenigsten gut klappen («deliberate practice»). Die besten unter den Experten sind jene, welche die meisten Stunden mit hartem Training von denjenigen Dingen zugebracht haben, die am schwierigsten sind (immer wieder das trainieren, was am schlechtesten funktioniert).

mern» und erst durch einen oder mehrere Auslöser (Trigger und Crosstrigger) im Zusammenhang mit zusätzlichen assoziierten Faktoren in Erscheinung treten und sich negativ auf einen ablaufenden Prozess auswirken. Reason [13] hat diesbezüglich den Begriff der Fehlerkette eingeführt.

Ein gutes System besitzt darum Sicherheitsschranken, die eine Fehlerentstehung verhindern bzw. unterbrechen sollen. Hierbei handelt es sich z.B. um regelmäßiges Briefing, Checklisten, Algorithmen oder Richtlinien, aber auch «cross-checking» zwischen einzelnen Teammitgliedern (Kasten 1). Standardisierte Abläufe senken die Fehlerwahrscheinlichkeit und erhöhen damit die Prozessqualität. Außerdem erhöhen sie die Vorhersagbarkeit eines Systems. Der eine Fehlerentwicklung auslösende Trigger (wie Ereignisse, eine Person oder die äußere Umgebung als assoziierter Faktor) ist häufig räumlich und zeitlich getrennt vom schlummernden latenten Fehler. $\mathrm{Zu}$ den assoziierten Faktoren gehört auch die menschliche Leistungsfähigkeit (Human Performance) mit allen Einschränkungen. Hierbei handelt es sich um ein vernetztes Geschehen, während monokausale Ereignisse selten sind [9, 10, 13-15].

In Bezug auf die Verteilung und Häufigkeit von Fehlern unterscheidet man die zufällige Verteilung von der systematischen bzw. der sporadischen Verteilung (zufällige, systematische bzw. sporadische Fehler). Sporadische Fehler lassen sich in ihrer Auftretenswahrscheinlichkeit nur schwer vorhersagen und haben zumeist sehr differente Ursachen. Gleiches gilt für die zufällig verteilten Fehler. Ganz im Gegensatz zu den systematischen Fehlern, die den größten Anteil ausmachen, sind sporadische und zufällig verteilte Fehler dem Fehlermanagement nur schwer zugänglich.

\section{Fehlerkette und Fehlerentstehung}

Die Redensart «Ein Fehler kommt selten allein» beschreibt ganz richtig, dass es sehr unwahrscheinlich ist, dass ein singulärer Fehler unmittelbar und direkt zu einem Zwischenfall wird. Vielmehr bedarf es neben dem eigentlichen Fehler einer Reihe begleitender Umstände, die sich zu einer Fehlerkette formieren.
Reason beschreibt in seiner Fehlertheorie einen trichterähnlichen Mechanismus vom auslösenden Fehlerereignis bis hin zum Zwischenfall. Im modernen Fehlermanagement finden wir die Ursache fehlerhafter klinischer Prozesse häufig bereits auf der organisatorischen Ebene. Strukturen der Organisationen sowie fehlerhaft etablierte Handlungsebenen und Verfahren spielen hierbei eine zentrale Rolle [13, 15].

Die unmittelbar zu bewältigende Akutsituation und das dafür notwendige Arbeitsumfeld sind ein weiteres Kettenglied. Um fehlerarme Handlungsweisen wahrscheinlich zu machen, muss die Umgebung sehr sorgfältig an die zu lösende Aufgabe und den Ausführenden adaptiert werden. Das notwendige Arbeitsumfeld ist ein wichtiges Glied von Fehlerketten.

Das handelnde Individuum steht als letztes Glied der Kette mit all seinen Stärken und Schwächen. Im klinischen Umfeld ist es häufig die letzte Instanz auf dem Weg zum Zwischenfall. Es ist/wäre unmittelbar in der Lage, Systemschwächen oder eingebaute Strukturfehler noch zu kompensieren oder aber selbst den latenten Fehler in einen aktiven Fehler zu transformieren, um somit die Fehlerflugbahn letztlich auszulösen.

\section{Fehlerinduzierende Bedingungen}

Eine Vielzahl der Umstände des jeweiligen Handlungsumfelds ist vom klinischen Personal nur wenig oder nicht beeinflussbar. Diese Umstände tragen aber in einem erheblichen Maß zur Fehlerhäufigkeit Einzelner bei. Die Bedeutung der einzelnen Umstände ist in Tabelle 2 gewichtet [15].

\section{Fehlervermeidung und Fehlerbehandlung}

«Der immer das macht, was er immer tut, wird immer zu dem Ergebnis kommen, zu dem er immer kommt» (nach [13]).

Wir sind in der operativen und interventionellen Akutmedizin im besonderen Maße verpflichtet, unser Arbeiten und Handeln auf immer neue Aspekte hin zu überprüfen und an neue Erfordernisse und Erkenntnisse anzupassen.

Diese Umstände erfordern in besonderem Maße Vorkehrungen, um das Eintreten von Fehlern so unwahrscheinlich wie möglich zu machen. Da aber in derart komplexen Systemen mit vielen unterschiedlichen Anwendern Fehler nie hundertprozentig ausgeschlossen werden können, müssen in einem weiteren Schritt Gegenmaßnahmen ergriffen werden, um die Folgen eingetretener Fehler so gering wie möglich zu halten.

\section{Standard Operating Procedures}

Für viele Risiko- und Hochrisikoarbeitsbereiche sind Standard Operating Procedures (SOPs) heutzutage eine Selbstverständlichkeit und in Notfallsituationen als entscheidende Handlungsanweisungen nicht mehr wegzudenken [7, 8, 16-20].

SOPs in der Medizin und insbesondere in der Akutmedizin zu etablieren ist ebenso zwingend wie schwierig. Viele Ärztinnen und Ärzte sehen in SOPs nicht eine gut indizierte Ar- 
Tab. 2. Bedeutung der einzelnen Umstände des Handlungsumfelds

\begin{tabular}{lll}
\hline Bedingung & Faktor $^{\mathrm{a}}$ & Kategorie \\
\hline Unbekannte, neue Aufgabe & mal 17 & Training, Erfahrung \\
Zeitknappheit & mal 11 & System, Umgebung \\
Schlechte Mensch-Maschine-Schnittstelle & mal 8 & Design \\
Irreversibilität von Fehlern & mal 8 & Design, System \\
Informations-Overload & mal 6 & Design, System \\
Fehleinschätzung des Risikos & mal 4 & Haltung, Ausbildung \\
Schlechtes Feedback aus dem System & mal 4 & Design \\
Unerfahrenheit (nicht fehlendes Training) & mal 3 & System, Erfahrung \\
Inadäquate Überprüfungen von Parametern & mal 3 & System, Erfahrung \\
Übermüdung, Schlafdefizit & mal 1,6 & Umgebung \\
Monotonie und Eintönigkeit & mal 1,1 & Umgebung \\
\hline
\end{tabular}

${ }^{\text {a }}$ Diese Zahl ist der Faktor, um den sich die Fehlerwahrscheinlichkeit einer bestimmten Tätigkeit erhöht, wenn die erwähnte Bedingung vorliegt. beitshilfe, sondern einen empfindlichen Eingriff in den Gestaltungsspielraum des persönlichen Entscheidungskorridors. Nicht selten wird Medizin noch primär als Heilkunst und nicht als Wissenschaft auf dem Boden erkenntnisbasierten Handelns betrachtet [19-21].

Im Notfall oder bei einer schweren Komplikation/Fehlerkette in der operativen und interventionellen Akutmedizin sind SOPs die Richtschnur des Handelns im Team. Ein Teammitglied fällt dann bereits als verantwortlicher Mitarbeiter in einer Akutsituation aus, wenn er in dieser Situation nicht das gleiche mentale Modell wie sein übriges (Ad-hoc-)Team hat. In dieser Situation dient das Gedächtnis gleichsam als Datenbasis, um die erforderlichen Entscheidungen herbeizuführen. Verwenden die Teammitglieder überwiegend die gleiche Datenbasis, sind folglich die Varianzen in den mentalen Modellen gering und dem Handeln entsprechend kongruent.

Dies ist im Notfall bei allen Teammitgliedern nicht nur erwünscht, sondern zwingend erforderlich. Solange alle Teammitglieder im OP die gleichen SOPs gespeichert und kognitiv zur Verfügung haben, sollte das mentale Modell des «Komplikationsmanagements» in weiten Teilen deckungsgleich sein $[8,16,17,20,22]$.

SOPs sind somit auch eine Art vorweggenommener Kommunikation und eine grundlegende Absprache über die Handlungsschritte, die als vorausgesetzt gelten können. Hieraus ergibt sich zwangsläufig, dass bei geplanten Abweichungen von SOPs alle Teammitglieder über diese Absicht unterrichtet werden müssen, um sie letztlich in die Lage zu versetzen, das mentale Modell und damit das individuelle Verhalten entsprechend abstimmen oder ihren Dissens artikulieren zu können [8, 22-24].

Gute Teammitglieder zeichnen sich unter anderem dadurch aus, dass sie Informationen über Abweichungen von SOPs kommunizieren und deren Handlungsgrundlage einfordern. Gute Teamleiter schaffen eine Arbeitsatmosphäre, die das Einfordern derartiger Informationen ermöglicht und stützt.

\section{Entscheidungsfindung und Motivation}

«Experten machen die gleichen Fehler wie der Rest von uns, lediglich unter anderen Umständen» [2].

In der Medizin hat die Fähigkeit der handelnden Personen, Entscheidungen schnell und richtig zu fällen, eine herausragende Bedeutung. Grundsätzlich unterscheidet sich dieser Mechanismus, der in der operativen Akutmedizin letztlich zu Entscheidungen führt, in keiner Weise vom Mechanismus anderer Entscheidungsträger im beruflichen Umfeld. Nicht selten findet in der Akutsituation die Entscheidungsfindung bei schlechter Informationslage und/oder fehlerhafter Datenlage statt.

In solchen Situationen werden die von den Sinnen erfassten Informationen vom Gehirn zu einem Modell verarbeitet (man spricht hier vom «mentalen Modell»). Es handelt sich somit um ein Bild der Wirklichkeit, wie wir sie uns in der jeweiligen Situation gerade vorstellen [2, 8].

Je weniger Informationen de facto für unsere Wahrnehmung zur Verfügung stehen, desto ungenauer ist das mentale Modell, das wir von der Wirklichkeit entwerfen. Ein grundlegendes Problem liegt darin begründet, dass das Gehirn fehlende Teile des mentalen Modells zu ergänzen versucht. Hierbei wird, gleichsam als Lagerstätte oder Datenbank von Schemata, auf das Langzeitgedächtnis zurückgegriffen. Diese Datenbasis, auf die im akuten Fall zur individuellen Entscheidungsfindung zurückgegriffen wird, ist natürlich nicht frei von Fehlern.

Darüber hinaus ist zu berücksichtigen, dass die Komplettierung fehlender Informationen durch frühere Erfahrungen aus dem Gedächtnis die Realität zwar treffen kann, jedoch nicht zwingend treffen muss. Zusätzlich ist von Bedeutung, dass jeder Mensch für die gleiche Situation oder vergleichbare Situationen unterschiedliche mentale Modelle entwickelt und abspeichert [8].

Die Affinität eines jeden Einzelnen von uns $\mathrm{zu}$ «seinen» mentalen Modellen ist sehr hoch. Jeder von uns ist bewusst oder unbewusst kontinuierlich dabei, «Erlebtes» durch die Einordnung in bereits bestehende mentale Modelle zu bewer- 
Tab. 3. CRM/TRM-Leitsätze nach Rall und Gaba (modifiziert nach $[9,26])$

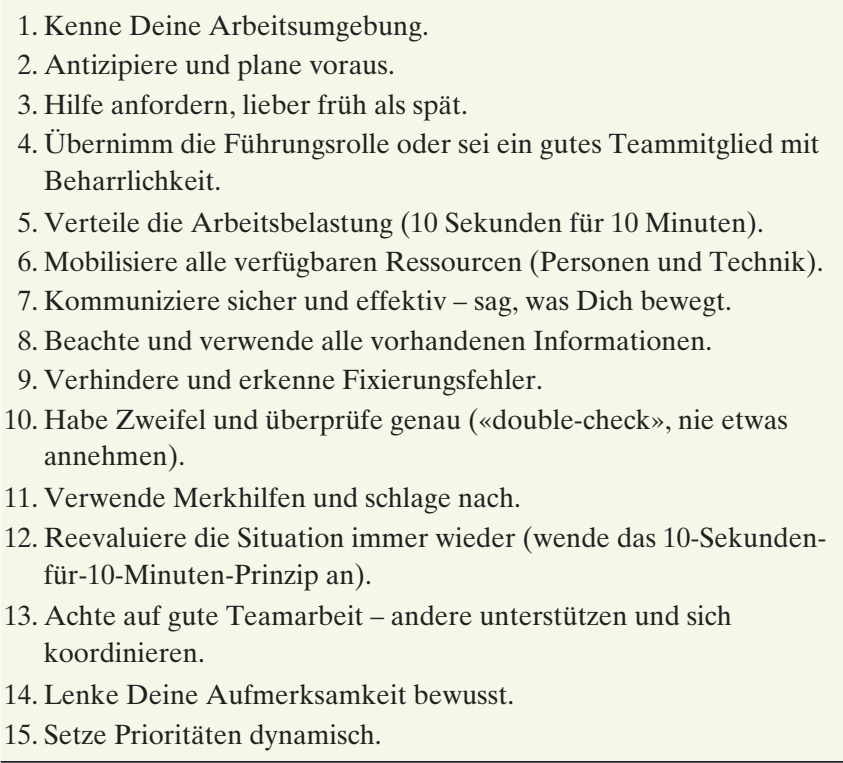

ten. Dies hat erhebliche Auswirkungen auf unsere Fehlervermeidungsstrategien.

Haben wir einmal eine falsche Entscheidung getroffen, so sind wir nur mühsam von unserem falschen Handlungsweg abzubringen, selbst wenn sich Erkenntnisse und Informationen verdichten, dass der gerade beschriebene Weg falsch ist. Im Gegenteil gehen wir sogar intuitiv davon aus, dass zur Problembewältigung verstärkte Anstrengungen - «mehr desselben» - notwendig sind. Für andere Problemlösungen, d.h. einen Strategiewechsel, haben wir im wahrsten Sinne des Wortes den Blick verloren [2, 13, 15, 25].

- Heuristische Entscheidungsfindung: Während man lange erwartet oder zumindest gehofft hatte, dass kognitive Entscheidungen ihre Ursache in der Abwicklung hochkomplexer Lösungsalgorithmen haben, weiß man heute, dass es sich der Mensch wesentlich leichter macht. Das grundlegende Muster zur Herbeiführung von Entscheidungen sind logische, zumeist vergleichsweise einfache Verknüpfungen, sogenannte «Heurismen».

- Repräsentativitätsregel: «Aus der Situation 1 folgt die Situation 2. Dies war beim letzten Mal ebenso.» - Frühere Erfahrungen suggerieren eine erhöhte statistische Wahrscheinlichkeit des Zusammenhangs.

- Verfügbarkeitsregel: Unsere zentrale Datenbasis, das Gedächtnis, speichert die mentalen Modelle und neu hinzugekommene Informationen gemäß ihrem zeitlichen Auftreten $\mathrm{ab}$.

Wir kennen die Unterscheidung in Kurzzeit- und Langzeitgedächtnis. Je länger eine Information, ein Sinneseindruck oder ein daraus resultierendes mentales Modell zurückliegt, desto größeren Aufwand bereiten dessen Suche und Verwendung.

Daraus resultiert, dass jeder von uns im Entscheidungsfall geneigt ist, sich eher an frischen, neuen Informationen und
Sinneseindrücken zu orientieren, als auf ältere oder bereits länger abgespeicherte mentale Modelle zuzugreifen. Somit ist von entscheidender Bedeutung, kritische Informationen stets aktuell zu halten, um im Entscheidungsfall niederschwellig darauf zugreifen zu können [8, 23].

\section{Die Motivation}

Eine erhebliche Anzahl von Fehlhandlungen, die zu Zwischenfällen führen, lässt sich im Rahmen der Fehleranalyse weder auf schlechtes Verfahrens- oder Handlungsdesign noch auf ungünstige Umgebungsbedingungen oder auf mangelndes Wissen bzw. mangelnde Fertigkeiten der handelnden Personen zurückführen. Hier stellt sich letztlich die Frage: Warum handelt ein Mitglied in einem klinischen Team so, wie es handelt?

Man fragt nach seiner/ihrer Motivation. Motivation in diesem Zusammenhang bezeichnet den Unterschied zwischen dem, was jemand «kann», und dem, was er/sie tatsächlich «macht».

Es gibt eine ganze Reihe zum Teil sehr unterschiedlicher Theorien darüber, was derartigen Handlungsweisen auf der Motivationsebene zugrunde liegen könnte. Einig sind sich diese Theorien darüber, dass es verschiedene Stufen von Wünschen und Bedürfnissen gibt, die verschieden stark danach verlangen, befriedigt zu werden.

In der Maslow'schen Theorie muss ein Bedürfnis umso eher befriedigt werden, je weiter unten es in der Pyramide angesiedelt ist. Erst danach spielen die übergeordneten Punkte eine relevante Rolle. Verschiedene Verhaltensweisen können dabei von mehreren Faktoren gleichzeitig und zum Teil auch gegensätzlich beeinflusst werden.

Die Motivation eines Menschen lässt sich durch eine entsprechende Gestaltung seiner Umgebung/seines Arbeitsumfelds stark beeinflussen [8].

\section{TRM-Training für den $O P$}

TRM beinhaltet Strategien und Verfahren, um die Wirkmechanismen des «Human Factor» zu erkennen und sie als Auslöser von Fehlerketten zu vermeiden. Es thematisiert Verhaltenscodes, welche die individuelle Handlungsexpertise und damit die Patientensicherheit durch präventive Maßnahmen und Bewältigungsstrategien in erfolgs- und prognosekritischen Situationen (Notfall, Zwischenfall) erhöhen können [9].

Crisis Resource Management (CRM) und TRM wurden von Gaba und Howard erstmals in Form des «Anesthesia Crisis Resource Managements» (ACRM) in die Medizin eingeführt $[4,26]$. ACRM ist aus bewährten Schulungskonzepten der Luft- und Raumfahrt abgeleitet und an die speziellen Belange der Medizin adaptiert [19].

Das von Gaba und Howard etablierte ACRM-Training am Simulator und die hieraus in Tabelle 3 dargestellten CRMPrinzipien haben sich mittlerweile, mit gewissen Varianten, weltweit de facto als Goldstandard beim Training mit Simulatoren und TRM-Trainingsmaßnahmen etabliert [9, 26]. 
Inzwischen besteht zunehmend die Erkenntnis, dass bei konsequenter Umsetzung der CRM/TRM-Leitsätze die überwiegende Mehrzahl der Fehler oder Zwischenfälle, deren Ursachen im Bereich «Human Factors» liegen (und das sind gemäß den Literaturbefunden mehr als $70 \%$ ), vermieden oder zumindest in der Auswirkung erheblich abgeschwächt werden könnte [2, 9, 27, 28].

Damit die TRM-Leitsätze nachhaltig und zielorientiert wirksam werden, sind eine intensive Beschäftigung, ein praktisches Training und ein routinierter Gebrauch erforderlich. Denn speziell in kritischen Situationen wird das Management der eigenen Fähigkeiten und des Teams besonders wichtig und damit für das Erkennen und Üben zugänglich [9, 27, 29].

\section{TRM-Fazit: Implementierung einer nachhaltigen Sicherheitskultur}

Die Implementierung und kontinuierliche Weiterentwicklung der Patientensicherheit im Bereich der «Human Factors» und die Anwendung der TRM-Leitsätze erfordert als Basis eine gute und breit akzeptierte Sicherheitskultur.

Hierbei können Initiativmaßnahmen wie regelmäßige realitätsnahe Simulationsteamtrainings und gut etablierte nichtpunitive Incident-Reporting-Systeme zu einer stetigen und messbaren Verbesserung der Sicherheitskultur beitragen.
TRM-orientiertes Teamtraining für die ganze Abteilung zeigt das Bekenntnis der medizinischen Führungsebene zu wirksamen Verbesserungen im Bereich «Human Factor» und TRM und verdeutlicht allen Teammitgliedern den Einfluss der «Human Factors» auf die individuelle Leistungsfähigkeit im Team und damit unmittelbar auf das Outcome der Patienten.

Bei derartigen Teamentwicklungsformaten wird für jedes Teammitglied unmittelbar erlebbar, dass

- jeder Fehler machen kann;

- berufliche Erfahrung keine Garantie für Fehlerfreiheit ist;

- auch relativ Unerfahrene sehr gute Teammitglieder sein können [9].

Diese Trainingsmaßnahmen induzieren einen intensiven inneren Zusammenhalt im Team, sorgen für deutlich mehr Verständnis für die Position der anderen Teammitglieder und fokussieren auf das gemeinsame Ziel «sichere Patientenbehandlung zählt» [9].

\section{Disclosure Statement}

Christian K. Lackner, Heinzpeter Moecke und Karin Burghofer sind Trainer für sogenannte «Human Factor»- und «Risk Management»-Trainingsformate für Kliniken und Rettungsdienste. Christian K. Lackner analysiert bei Drees und Sommer Kliniken bezüglich Risikoprofilen.

\section{Literatur}

1 Burghofer K, Ruchholtz S, Seekamp A, Lackner CK: Kommunikation am Notfallort. Notfallmedizin up2date 2008;3:121-132.

2 Burghofer K, Lackner CK: Die Safe:Trac-Kursreihe der DGU zur Patientensicherheit. Unfallchirurg 2009;112:753-755.

3 Helmreich R: On error management: lessons from aviation. Br Med J 2000;320:781-785.

4 Howard SK, Gaba DM, Fish KJ, et al: Anesthesia crisis resource management training: teaching anesthesiologists to handle critical incidents. Aviat Space Environ Med 1992;63:763-770.

5 Institute of Medicine: To Err Is Human: Building a Safer Health System. Washington, DC, National Academy Press, 1999.

-6 Lackner CK, Burghofer K: Dream Teams are made - not born! Notfall Rettungsmed 2010;13: 347-348.

7 Aktionsbündnis Patientensicherheit: Agenda Patientensicherheit 2011. www.aktionsbuendnis-patien tensicherheit.de/apsside/Agenda_2011_mit_Titelblatt.pdf.

8 Burghofer K, Lackner CK: Risiko- und Fehlermanagement; in Wirth C, Mutschler W, Bischoff $\mathrm{H}$ et al. (Hrsg): Komplikationen in Orthopädie und Unfallchirurgie. Stuttgart, Thieme, 2009, pp 32-49.

9 Rall M, Lackner CK: Crisis Resource Management (CRM) - der Faktor Mensch in der Akutmedizin. Notfall Rettungsmed 2010;13:349-356.

10 Scheppokat K: Fehler in der Medizin. Anfälligkeit komplexer Systeme. Dtsch Arztebl 2004;101:A998A999.
11 Lackner CK, Hoffmann G, Burghofer K: Nameblame-shame. Mitarbeiterführung auf dem Weg zur Sicherheitskultur. Berlin Medical 2005;4:8-9.

12 Sexton J, Thomas E, Helmreich R: Error, stress, and teamwork in medicine and aviation: crosssectional surveys. Br Med J 2000;320:745-749.

13 Reason JT: Human Error. Cambridge, Cambridge University Press, 1990.

14 Schulz von Thun F: Miteinander reden. Störungen und Klärungen. Rowohlt, Reinbek, 1981.

15 Reason J: Managing the Risks of Organizational Accidents. Aldershot, Ashgate, 1997.

16 Gawande A, Zinner M, Studdert D, Brennan T: Analysis of errors reported by surgeons at three teaching hospitals. Surgery 2003;133:614-621.

17 Haynes AB, Weiser TG, Berry WR, et al: A surgical safety checklist to reduce morbidity and mortality in a global population. N Engl J Med 2009;360: 491-499.

18 Thomas E, Studdert D, Burstin H, et al: Incidence and types of adverse events and negligent care in Utah and Colorado. Med Care 2000;38:247-249.

19 Wiener E, Kanki B, Helmreich R: Cockpit Resource Management. San Diego, Academic Press, 1993.

20 World Health Organization (WHO): WHO Surgical Safety Checklist. 2008. www.who.int/patientsafety/safesurgery/en.

21 Haynes A, Weiser T, Berry W, et al: A surgical safety checklist to reduce morbidity and mortality in a global population. N Engl J Med 2009;360:491499.
22 Rall M, Dieckmann P: Prävention und Management von kritischen Ereignissen durch Crisis Resource Management (CRM). Minim Invasive Chir 2005;14:31-38.

23 Burghofer K, Lackner CK: Kommunikation - Risikofaktor in der Akutmedizin. Notfall Rettungsmed 2010;13:347-348.

24 Rall M: Erhöhung der Patientensicherheit durch Crisis Resource Management (CRM) Training. J Anästh Intensivbehandl 2004;2:98-104.

25 Watzlawick P, Beavin J, Jackson D: Menschliche Kommunikation - Formen, Paradoxien, Störungen. Huber, Bern, 1971.

26 Rall M, Gaba DM: Human performance and patient safety; in Miller RD (ed): Miller's Anesthesia. Philadelphia, Elsevier Churchhill Livingstone, 2009, pp 93-150.

27 Draycott TJ, Crofts JF, Ash JP, et al: Improving neonatal outcome through practical shoulder dystocia training. Obstet Gynecol 2008;112:14-20.

28 Risser D, Rice M, Salisbury M, et al: The potential of improved teamwork to reduce medical errors in the emergency department. The MedTeams Research Consortium. Ann Emerg Med 1999;34:373383.

29 Hunt EA, Walker AR, Shaffner DH, et al: Simulation of in-hospital pediatric medical emergencies and cardiopulmonary arrests: highlighting the importance of the first 5 minutes. Pediatrics 2008;121: e34-e43. 